\title{
Block-crossed arrays with applications to robust design
}

\author{
Yue Fang ${ }^{\mathrm{a}, *}$, Bin Zhou ${ }^{\mathrm{b}}$ \\ ${ }^{a}$ Lundquist College of Business, 1208 University of Oregon, Eugene, OR 97403, USA \\ ${ }^{\mathrm{b}}$ Sloan School of Management, Massachusetts Institute of Technology, Cambridge, MA 02139, USA
}

Received 6 March 1997; accepted 3 March 1998

\begin{abstract}
In this paper we develop a class of two-level designs that allow orthogonal estimation of certain main effects and two-factor interactions when other main effects and two-factor interactions are present but not of interest. The designs can be easily generated using block-crossed arrays. The application is clearly to settings in which one might apply either Taguchi's crossed arrays or single-array designs suggested by many people as a better alternative to Taguchi's methods. Our designs have certain properties, the most important being the flexibility and a reduction, in many cases, in the number of runs over both Taguchi's designs and single orthogonal arrays. (C) 1998 Published by Elsevier Science B.V. All rights reserved.
\end{abstract}

AMS classification: $62 \mathrm{~K} 99$

Keywords: Fractional factorial designs; Screening analysis; Orthogonal arrays; Taguchi crossed arrays

\section{Introduction}

In robust product designs, main effects of design factors and interactions between design and environment factors are the most important to be studied (see e.g., Wu et al., 1990; Shoemaker et al., 1991). If certain interactions, such as interactions among design factors, are not negligible, the experimental design should, therefore, be chosen so that the main effects of design factors as well as the interactions between design and environment factors can be estimated without being confounded with interactions among design factors.

Taguchi (see Taguchi, 1986, among many others) recommended the use of inner and outer orthogonal arrays to find design factors insensitive to the variation caused by uncontrolled environmental sources. For each experimental run in the inner array where the primary design factors are varied over a certain range, another outer array of runs is conducted, varying environment factors that may affect the product's performance. Most

*Corresponding author. Fax: +1 5413463341 ; e-mail: yfang@darkwing.uoregon.edu. 
of Taguchi's inner and outer arrays are two-, three- or mixed-level classic fractional factorial designs (Box et al., 1987; Kacker et al., 1991). In some of Taguchi's designs, the inner orthogonal arrays are highly fractional designs, while the main effects of design factors are confounding with two-factor interactions. If interactions among design factors do indeed exist, analysis results often are confused and misleading (Hunter, 1985; Kackar, 1985; Box, 1988). A few runs of a follow-up confirmation experiment is often recommended in practice when high-fractional design is used. But the confirmation experiment cannot detect significant design factors that fail to be found in the first place (Logothetis and Wynn, 1989; Lucas, 1994). In order to estimate the main effects of design factors free of two-factor interactions, a design with resolution IV or higher is required in the inner array. Because of the cross-array arrangement, the total number of experimental runs is the product of the size of inner and outer arrays. Even in the screening analysis, for a system with many design and environment factors, the cross-array arrangement often involves a large amount of experimental work.

Many people (see e.g., Box et al., 1987; Bisgaard, 1989) suggested the use of a single array instead of Taguchi's crossed arrays. The use of a single orthogonal array can sometimes reduce the experimental runs and simplify the confounding structure, especially to free the main effects of design factors of two-factor interactions. The single array is based on traditional fractional factorial designs with the run size of a power of two. It is constructed either by choosing the design generators carefully so that the effects which are desired are not confounded or by assuming that some two-factor interactions are negligible (see e.g., Wu and Chen, 1992). Since the singlearray experiment plan is based on the detailed analysis of the confounding structure of fractional factorial designs with low resolutions, an efficient design can be obtained for only certain combinations of the numbers of design and environment factors (Whitwell and Morbey, 1961; Addelman, 1962; Box and Jones, 1990a, b).

In this paper, we develop a block-crossed array strategy, to construct designs providing orthogonal estimates of most important effects in the screen stage of the robust design of experiment. The objective of interest is to estimate main effects of design factors and interactions among design and environment factors. Assume that all three or higher order interactions are zeros, the designs can be used in estimating orthogonally main effects of design factors, as well as design $\times$ environment interactions free of other main and two-factor interaction effects. The proposed plan is a systematic method for constructing a series of desired designs for any size experiment system. It often requires fewer runs than Taguchi's crossed arrays and traditional single-array designs. The paper is organized as follows. In Section 2 block-crossed arrays are introduced with an illustrated example. Section 3 discusses the run sizes and the alias properties of the proposed designs. We summarize the results in Section 4.

\section{Block-crossed array approach}

We consider designs with two levels, + and - . For any column vector $\bar{A}$, denote $+\bar{A}=\bar{A}$ and $-\bar{A}$ to be the vector with all signs reversed in $\bar{A}$. Let $\bar{I}$ be the vector of all 
+ elements. Then $-\bar{I}$ is the vector of all - elements. Suppose that there are $n$ design factors and $m$ environment factors. The step by step procedure for the construction of a block-crossed array is as follows.

Block-crossed array procedure.

(1) Construct an orthogonal design with a resolution at least III for $n$ design factors. Denote this design matrix as $X$ with the run size $x$.

(2) If the design obtained in Step 1 has resolution IV or higher, construct an orthogonal resolution III design with run size $z$ for $m$ environment factors. Otherwise, construct an orthogonal resolution III design for $m+1$ factors. Denote the matrix as $Z$ and the $i$ th column of $Z$ as $\bar{Z}_{i}$.

(3) Obtain the block-crossed array for these $n+m$ factors.

Case 1: The design obtained in Step 1 has resolution IV or higher. In this case, assign $n$ design factors to the columns of $X$ with replacement of + and - by small block $+\bar{I}_{z \times 1}$ or $-\bar{I}_{z \times 1}$, respectively; assign the $i$ th environment factor to a vector with $x$ small blocks $\bar{Z}_{i}$.

Case 2: The design obtained in Step 1 has resolution III. Assume that $q$ factors can be accommodated in this $x$-run design such that the design for these $q$ factors is of resolution IV or higher. Assume that the first $q$ columns in $X$ are corresponding to these $q$ factors. In this case, assign the first $q$ design and $m$ environment factors the same way as in case 1; assign the remaining $n-q$ design factors to vectors with $x$ small blocks $\bar{Z}_{m+1}$ or $-\bar{Z}_{m+1}$ with the signs corresponding to the last $n-q$ columns of $X$.

The block-crossed array is essentially a crossed combination of two resolution III arrays. By the way it is constructed, the design provides orthogonal estimation of main design factors as well as design $\times$ environment interactions free from other main and two-factor interaction effects. The proof of the orthogonality properties in block-crossed arrays is outlined in Appendix. It is important to note that the number of runs of both arrays $X$ and $Z$ are not necessarily to be a power of two. Therefore, many orthogonal resolution III designs can serve as candidates in Step 1 and 2 in the block-crossed array procedure. We will use Plackett-Burman (1946) designs and fractional factorial designs with a run size of a power of two, to demonstrate the block-crossed method in our discussion.

The Plackett-Burman designs are saturated resolution III designs. The advantage of the use of Plackett-Burman designs is, in some cases, the potential reduction in the number of runs. On the other hand, nongeometric properties of Plackett-Burman designs cause complicated confounding structures, especially when the run size is relatively large, say, more than 12. In contrast to Plackett-Burman designs, fractional factorial designs with a run size of power two are not, in general, saturated designs. However, fractional factorial designs with a run size of power two have simpler confounding structures among main and interaction effects, which provide attractive projection properties. 
Table 1

\begin{tabular}{llllll}
$2_{\mathrm{III}}^{6-3}$ & array $X$ & & & \\
\hline $\mathrm{A}$ & $\mathrm{B}$ & $\mathrm{C}$ & $\mathrm{D}=\mathrm{ABC}$ & $\mathrm{E}=\mathrm{AB}$ & $\mathrm{F}=\mathrm{AC}$ \\
\hline+ & + & + & + & + & + \\
+ & + & - & - & + & - \\
- & + & + & - & - & - \\
+ & - & + & - & - & + \\
+ & - & - & + & - & - \\
- & - & + & + & + & - \\
- & + & - & + & - & + \\
- & - & - & - & + & + \\
\hline
\end{tabular}

Table 2

Block-crossed array

\begin{tabular}{llllllllllll}
\hline $\mathrm{A}$ & $\mathrm{B}$ & $\mathrm{C}$ & $\mathrm{D}$ & $\mathrm{E}$ & $\mathrm{F}$ & $\mathrm{G}$ & $\mathrm{H}$ & $\mathrm{J}$ & $\mathrm{K}$ & $\mathrm{L}$ & $\mathrm{M}$ \\
\hline$+\bar{I}$ & $+\bar{I}$ & $+\bar{I}$ & $+\bar{I}$ & $+\bar{R}$ & $+\bar{R}$ & $\bar{G}$ & $\bar{H}$ & $\bar{J}$ & $\bar{K}$ & $\bar{L}$ & $\bar{M}$ \\
$+\bar{I}$ & $+\bar{I}$ & $-\bar{I}$ & $-\bar{I}$ & $+\bar{R}$ & $-\bar{R}$ & $\bar{G}$ & $\bar{H}$ & $\bar{J}$ & $\bar{K}$ & $\bar{L}$ & $\bar{M}$ \\
$-\bar{I}$ & $+\bar{I}$ & $+\bar{I}$ & $-\bar{I}$ & $-\bar{R}$ & $-\bar{R}$ & $\bar{G}$ & $\bar{H}$ & $\bar{J}$ & $\bar{K}$ & $\bar{L}$ & $\bar{M}$ \\
$+\bar{I}$ & $-\bar{I}$ & $+\bar{I}$ & $-\bar{I}$ & $-\bar{R}$ & $+\bar{R}$ & $\bar{G}$ & $\bar{H}$ & $\bar{J}$ & $\bar{K}$ & $\bar{L}$ & $\bar{M}$ \\
$+\bar{I}$ & $-\bar{I}$ & $-\bar{I}$ & $+\bar{I}$ & $-\bar{R}$ & $-\bar{R}$ & $\bar{G}$ & $\bar{H}$ & $\bar{J}$ & $\bar{K}$ & $\bar{L}$ & $\bar{M}$ \\
$-\bar{I}$ & $-\bar{I}$ & $+\bar{I}$ & $+\bar{I}$ & $+\bar{R}$ & $-\bar{R}$ & $\bar{G}$ & $\bar{H}$ & $\bar{J}$ & $\bar{K}$ & $\bar{L}$ & $\bar{M}$ \\
$-\bar{I}$ & $+\bar{I}$ & $-\bar{I}$ & $+\bar{I}$ & $-\bar{R}$ & $+\bar{R}$ & $\bar{G}$ & $\bar{H}$ & $\bar{J}$ & $\bar{K}$ & $\bar{L}$ & $\bar{M}$ \\
$-\bar{I}$ & $-\bar{I}$ & $-\bar{I}$ & $-\bar{I}$ & $+\bar{R}$ & $+\bar{R}$ & $\bar{G}$ & $\bar{H}$ & $\bar{J}$ & $\bar{K}$ & $\bar{L}$ & $\bar{M}$ \\
\hline
\end{tabular}

Before we discuss more properties of the proposed design, a simple example is appropriate to demonstrate the method.

Example. Consider six design and six environment factors: i.e., $n=6$ and $m=6$. Let $\mathrm{A}, \mathrm{B}, \mathrm{C}, \mathrm{D}, \mathrm{E}$ and $\mathrm{F}$ refer to six design factors and $\mathrm{G}, \mathrm{H}, \mathrm{J}, \mathrm{K}, \mathrm{L}$ and $\mathrm{M}$ refer to six environment factors. Suppose that we use $2_{\text {III }}^{6-3}$ design for six design factors. The design matrix of $X$ is given in Table 1 . The maximal integer $q$ is four $(q=4)$ in this example: i.e., if we only consider factors A, B, C and D, the design is of resolution IV. Because the design $2_{\mathrm{III}}^{6-3}$ is of resolution III, we need to construct a resolution III design for seven $(m+1=7)$ factors. We may use either an eight-run Plackett-Burman design or the design $2_{\mathrm{III}}^{7-4}$ to obtain the matrix $Z$ for six environment factors. Let $\bar{R}$ be the last column of $Z$. Denote the first six columns of $Z$ as $\bar{G}, \ldots, \bar{M}$, respectively. The block-crossed design matrix is given in Table 2 with total number of runs $64(8 \times 8)$.

\section{Run size and alias properties}

To decide the minimal run size in block-crossed arrays, we first give a brief review of a minimum number of runs for orthogonal resolution III designs. The run size for block-crossed arrays is then the product of two resolution III designs unless the array 
obtained in Step 1 of the block-crossed array procedure in Section 2 has resolution IV or higher.

In order to have block-crossed arrays with the minimal run size, Plackett-Burman designs should be used in constructing both arrays $X$ and $Z$. Plackett-Burman designs which are based on the Hadamard matrices are saturated orthogonal arrays. However, they only exist when the number of factors is a module of four. When the number of factors investigated is not a module of four, we need to use a larger available PlackettBurman design. For example, the minimal number of runs required in orthogonal resolution III designs for the number of factors from 4 to 15 are $8,8,8,8,12,12,12,12,16$, 16,16 , and 16, respectively. When the run size of a Plackett-Burman design is a power of two, it is identical to the traditional fractional factorial design with a run size of power two (see Example in Section 2 and the discussion in Montgomery, 1991).

To compare the run size of block-crossed arrays with single array plans, let us consider the example with six design and six environment factors in Section 2. The minimal run size of orthogonal resolution III design for six factor is eight. Hence, the number of runs required in the block-crossed array is 64. If we assume that interactions among design factors are not present, a single array plan suggested by Box and Jones (1990a) for six design and six environment factors requires the same number of runs (64) as the block-crossed array. However, this single array has resolution III. If interactions are significant, some main design factors are confounding with two-factor interactions $(\mathrm{D}=\mathrm{AB}, \mathrm{E}=\mathrm{AC}$, and $\mathrm{F}=\mathrm{BC})$. A single array with higher resolution (IV) for six design and six environment factors requires $2^{7}$ runs (Box and Jones, 1990b). The $2^{7}$-run array doubles the run size and the benefit is the power to estimate interactions among design factors by choosing the design such that, in the defining relation, the only factors that appear in any word of length four are environment factors. On the other hand, interactions among design factors may confound with each other in the block-crossed arrays. For example, $\mathrm{AD}=\mathrm{BC}=\mathrm{EF}$ in the example given in Section 2 .

If we focus only on the left-hand-side half of the block-crossed array, namely, the array for design factors, the design is of resolution IV but not of resolution $\mathrm{V}$. On the other hand, the right-hand-side half of the block-crossed design, which is for environment factors, is formed by folding over a resolution III design simply with the same sign (not with the sign reversed). There is no gain in terms of resolution in the right-hand-side half of the block-crossed array and the main environment effects are confounding with their two-factor interactions. It is important to emphasize that the block-cross arrays will become valuable tools only if one is interested in estimating main design and design $\times$ environment effects when other interactions are significant but not of interest.

\section{Concluding remarks}

The motivation of our work is to use robust designs to screen a large number of design factors. By introducing a block-cross array strategy, a series of designs are 
developed. The proposed designs provide orthogonal estimations of main effects of design factors, as well as design $\times$ environment interactions, free of other main effects and two-factor interactions. The method can generate designs for any size system. The number of runs required in the block-crossed array designs are, in general, less than those required by Taguchi's crossed arrays and single orthogonal array designs.

\section{Acknowledgements}

This research was partially sponsored by Leaders For Manufacturing program at MIT. We thank Roy Welsch and anonymous referees for their helpful comments.

\section{Appendix A. Orthogonality properties in block-crossed arrays}

Assume that the expected response $E(y)$ can be described by the model

$$
E(y)=\mu+\sum_{i=1}^{n} \alpha_{i} x_{i}+\sum_{j=1}^{m} \beta_{j} z_{j}+\sum_{i, j} \gamma_{i j} x_{i} z_{j}+\sum_{i, k} \delta_{i k} x_{i} x_{k}+\sum_{j, l} \eta_{j l} z_{j} z_{l},
$$

where $x_{i} \mathrm{~s}$ and $z_{j} \mathrm{~s}$ are design and environment factors, respectively. Denote the blockcrossed array as $\mathscr{B}=\left[B_{1}, \ldots, B_{n}, B_{n+1}, \ldots, B_{n+m}\right]$, where the first $n$ column vectors are design factors and the remaining $m$ column vectors are environment factors. If the design obtained in Step 1 has resolution IV or higher (Case 1), it is easy to verify that 1. $B_{i}^{\mathrm{T}} B_{j}=0$ for $i, j \leqslant n, i \neq j$ or $i \leqslant n$ and $j>n$;

2. $B_{i}(i \leqslant n)$ is orthogonal to all two-factor interactions;

3. interaction $B_{i} B_{j}(i \leqslant n$ and $j>n)$ is orthogonal to $B_{k}(k>n)$;

4. interaction $B_{i} B_{j}(i \leqslant n$ and $j>n)$ is orthogonal to other interactions.

If there exists a resolution III design in Step 1 (Case 2), we only need to verify that $B_{j}(j=q+1, \ldots, n)$ is orthogonal to all other main factors and two-factor interactions. This is true because the last column in $Z$ is orthogonal to all other columns in $Z$.

\section{References}

Addelman, S., 1962. Symmetrical and asymmetrical fractional factorial plans. Technometrics 4, 47-58.

Bisgaard, S., 1989. Quality engineering and Taguchi methods: a perspective. Target, October, 13-19.

Box, G.E.P., 1988. Signal-to-noise ratios, performance criteria, and transformations. Technometrics 30, 1-17.

Box, G.E.P., Bisgaard, S., Fung, C., 1987. An explanation and critique of Taguchi's contribution to quality engineering. Qual. Reliab. Eng. Internat. 4, 123-131.

Box, G.E.P., Jones, S.P., 1990a. Robust product designs, part I: first-order models with design $\times$ environment interactions. Report No. 62, Center for Quality and Productivity Improvement, University of Wisconsin, Madison.

Box, G.E.P., Jones, S.P., 1990b. Robust product designs, part II: Second-order models. Report No. 63, Center for Quality and Productivity Improvement, University of Wisconsin, Madison.

Hunter, J.S., 1985. Statistical design applied to product design. J. Qual. Technol. 17, 210-221.

Kackar, R.N., 1985. Off-line quality control, parameter design, and the Taguchi method. J. Qual. Technol. 17, 176-209. 
Kackar, R.N., Lagergren, E.S., Filliben, J.J., 1991. Taguchi's fixed-element arrays are fractional factorials. J. Qual. Technol. 23, 107-116.

Logothetis, N., Wynn, H.P., 1989. Quality Through Design: Experimental Design, Off-Line Quality Control and Taguchi's Contributions. Clarendon Press, Oxford.

Lucas, J.M., 1994. How to achieve a robust process using response surface methodology. J. Qual. Technol. 26, 248-260.

Montgomery, D.C., 1991. Design and analysis of experiments. Wiley, New York.

Plackett, R.L., Burman, J.P., 1946. The design of optimum multifactorial experiments. Biometrika 33, $305-325$.

Shoemaker, A., Tsui, K.L., Wu, C.F.J., 1991. Economical experimentation methods for robust design. Technometrics 33, 415-427.

Taguchi, G., 1986. Introduction to Quality Engineering: Designing Quality Into Products and Processes. Kraus International Publications, New York.

Whitwell, J.C., Morbey, G.K., 1961. Reduced designs of resolution five. Technometrics 3, 459-477.

Wu, C.F.J., Mao, S.S., Ma, F.S., 1990. SEL: A search method based on orthogonal arrays. In: Ghosh S. (Ed.), Statistical Design and Analysis of Industrial Experiments. Marcel Dekker, New York.

Wu, C.F.J., Chen, Y., 1992. A graph-aided method for planning two-level experiments when certain interactions are important. Technometrics 34, 162-175. 